\title{
Fetale Alkoholspektrumstörung - Diagnose und frühe Förderung
}

Dr. med. Dipl.-Psych. Mirjam N. Landgraf, München

\begin{abstract}
Schätzungen zufolge haben mindestens 1\% aller Kinder und Jugendlichen in Deutschland eine Fetale Alkoholspektrumstörung (FASD). Die irreversible Gehirnschädigung ist auf den Alkoholkonsum der Mutter während der Schwangerschaft zurückzuführen. Hebammen kommt bei der Prävention eine Schlüsselrolle zu. Sie können über die Risiken des Trinkens aufklären und dazu beitragen, dass Kinder frühestmöglich diagnostiziert und adäquat gefördert werden.
\end{abstract}

\section{Prävalenz}

Aus internationalen Studien lässt sich ableiten, dass ein wiederholter Alkoholkonsum oder ein mindestens einmalig auftretendes Rauschtrinken (Binge-Drinking) während der Schwangerschaft das Risiko der Entwicklung eines FASD birgt. Doch rund ein Drittel der schwangeren Frauen konsumiert Alkohol: Laut der Studie „Gesundheit in Deutschland Aktuell“ [1] zeigen ca. 20\% der schwangeren Frauen einen moderaten und ca. $8 \%$ einen riskanten Alkoholkonsum.

Das sogenannte Binge-Drinking (mindestens 5 alkoholische Getränke zu einer Gelegenheit wie Geburtstag, Silvester, Weihnachten o.ä.) unternehmen 12\% der Frauen seltener als einmal pro Monat, knapp 4\% einmal pro Monat und $0,1 \%$ jede Woche. Dieses Binge-Drininkg, bei dem es kurzfristig zu hohen Alkohol-Peeks im Blut der Mutter kommt, wirkt sich besonders schädlich aufs kindliche Outcome aus.

Denn während die mütterliche Leber den Alkohol binnen Stunden abzubauen vermag, wird dieser beim Embryo oder Fetus in den Intrazellularräumen gespeichert und kann durch die unreife Leber nicht abgebaut werden. So bleibt der Alkohol - Expertenschätzungen zufolge - zehnmal länger im Körper des Fetus als in dem der Mutter.

Studien aus Italien gehen davon aus, dass mindestens 2-5\% aller Schulkinder eine Fetale Alkoholspektrumstörung aufweisen [6][7]. Wegen der strikteren diagnostischen Kriterien in Deutschland schätzen Experten hierzulande, dass 1\% aller Kinder, also ca. 130.000 Kinder, betroffen sind. Hochgerechnet auf die Gesamtbevölkerung würden in der Bundesrepublik ca. 0,8 Millionen Menschen mit FASD leben - eine höhere Prävalenz als z.B. für Down-Syndrom oder Cerebralprarese. Viele der Betroffenen werden nicht diagnostiziert, obwohl sie typische Auffälligkeiten aufweisen. Dadurch erhalten sie keinen Zugang zu adäquater Versorgung.

\section{Formen der FASD}

Alkoholkonsum der Mutter während der Schwangerschaft ist häufig und kann das ungeborene Kind gravierend schädigen. Die intrauterinen Schädigungen des kindlichen Gehirns sind biologisch irreversibel und führen zu lebenslangen, vielfältigen Einschränkungen in Entwicklung, Kognition, Verhalten und Alltagsfertigkeiten. Die Belastung der betroffenen Menschen, ihrer Familien und ihrer Umgebung ist immens.

Schädigungen, die durch intrauterine Alkoholexposition hervorgerufen sind, werden unter dem Oberbegriff Fetale Alkoholspektrumstörungen (FASD - fetal alcohol spectrum disorders) zusammengefasst.

Zu den Fetalen Alkoholspektrumstörungen gehören

- das Vollbild Fetales Alkoholsyndrom (FAS - fetal alcohol syndrome)

- das partielle Fetale Alkoholsyndrom (pFAS - partial fetal alcohol syndrome)

- die alkoholbedingte entwicklungsneurologische Störung (ARND - alcohol related neurodevelopmental disorder)

\section{Risikofaktoren}

Die Kenntnis von Risikofaktoren für den Alkoholkonsum der Mutter während der Schwangerschaft und für die Entstehung einer FASD ist zur frühzeitigen Aufklärung und Intervention notwendig.

\section{Risikofaktoren für Alkoholkonsum der Schwangeren}

Ältere alleinstehende Frauen ohne Migrationshintergrund, mit hohem sozio-ökonomischem Status, deren Partner oder enge Bezugspersonen Alkohol trinken, die rauchen oder illegale Drogen nehmen, die unbeabsichtigt schwanger werden oder die psychisch erkrankt sind, haben ein erhöhtes Risiko, in der Schwangerschaft 
Alkohol zu konsumieren [2]. Weitere Risikofaktoren für Alkoholkonsum der Schwangeren sind:

- Alter > 30 Jahre (Binge-Drinking < 27 Jahre)

- Beginn von Alkoholkonsum in einem frühen Lebensalter

- Alkoholkonsum und insbesondere Binge-Drinking vor der Schwangerschaft

- vorherige Therapie wegen Alkoholproblemen

- Schwangerschaftsbesonderheiten (z.B. ungeplante oder ungewollte Schwangerschaft, selten oder spät begonnene pränatale Vorsorge)

- geringe soziale Unterstützung

- stattgefundene oder aktuelle körperliche Misshandlung oder sexueller Missbrauch durch Partner oder Fremden

- psychische und psychiatrische Störungen (z.B. Depression, Angst- oder Panikstörung, sexuelle Funktionsstörung)

\section{Risikofaktoren für Entwicklung eines FASD}

Das Risiko für das pränatal Alkohol exponierte Kind, an FASD zu erkranken, steigt mit der Höhe und der Dauer des mütterlichen Alkoholkonsums vor und während der Schwangerschaft, mit dem mütterlichen Alter, mit einer Mangelernährung und mit dem Beikonsum anderer Drogen. Ebenso können der väterliche Alkoholkonsum, geburtshilfliche Komplikationen und mögliche genetische Prädispositionen eine Rolle spielen. [2].

Das Zusammenspiel und die Gewichtung der verschiedenen Risikofaktoren ist komplex. Warum es vorkommt, dass bei zwei Frauen, die in der Schwangerschaft Alkohol trinken, eine ein gesundes Kind bekommen kann,

TIPP

Empfehlung für die Beratung von Schwangeren Wer Frauen in der Schwangerschaft begleitet, sollte die Risikofaktoren kennen. Beobachten Sie die Frauen, ihre Partner und die anderen Kinder. Fragen Sie sensibel nach dem Alkoholkonsumverhalten in der Familie. Auch psychische Faktoren wie Missbrauchserfahrungen sind ein sehr wichtiges Thema, das sensibel angesprochen werden sollte. Diese extreme psychische Belastung muss auch in der weiteren Begleitung der Schwangerschaft berücksichtigt werden. Das Trauma kann zusammen mit einem weiteren Stressor, der vielleicht in der Schwangerschaft liegt, dazu führen, dass die werdende Mutter trinkt. Unter Depressionen oder anderen psychiatrischen Erkrankungen leidende Frauen haben ein erhöhtes Risiko für Alkoholkonsum in der Schwangerschaft. Sie sprechen vielleicht nicht über ihre Erkrankung und sind eventuell auch gar nicht in Therapie. Daher trinken sie, um depressive Gedanken loszuwerden. 


\section{INFO}

\section{Kein Alkohol in der Schwangerschaft}

Da Alkohol zu den tolerierten Rauschmitteln zählt und gesellschaftlich vermeintlich „einfach dazugehört“, wird häufig die Frage gestellt: „Wie viel darf ich denn in der Schwangerschaft trinken?" Die Antwort ist kompromisslos:

In der Schwangerschaft gibt es keinen Zeitpunkt und keine Menge Alkohol, der oder die erwiesenermaßen unschädlich ist.

Diese häufig gestellte Frage zeigt das Ausmaß der Unkenntnis in der Bevölkerung. Niemand würde das Risiko eingehen, ungeschützten Geschlechtsverkehr zu haben, wenn er sich bei einem Partner mit HIV infizieren könnte. Und niemand würde fragen: „Wie hoch ist die Wahrscheinlichkeit einer Infektion, falls ich es doch tue?"

Alkoholkonsum ist zu jedem Zeitpunkt in der Schwangerschaft riskant: Alkohol ist ein direkt wirksames Zellgift. Mütterlicher Alkoholkonsum zerstört Zellen im Gehirn des Fetus oder verhindert deren Entwicklung. Die Frau muss für sich selbst entscheiden: „Ich möchte dieses Risiko für mein Kind nicht eingehen und deswegen trinke ich nicht." Ein motivierender oder selbst abstinenter Partner kann sie dabei unterstützen.

während das Kind der anderen Frau ein FASD entwickelt, ist noch nicht abschließend geklärt.

Nach aktueller Studienlage bekommen auch Kinder von Drogenabhängigen (Heroin, Amphetamine, andere Substitutionsmittel) nach Überstehen des neonatalen Entzugssyndroms Folgeprobleme der Drogenexposition im Mutterleib. Aber diese sind, bezugnehmend auf die aktuelle Studienlage, nicht vergleichbar mit den Schädigungen durch Alkoholkonsum während der Schwangerschaft. Alkohol ist scheinbar die Substanz, die Kinder im Langzeitverlauf am meisten schädigt. Dahingehend sollten Frauen aufgeklärt werden.

Weitere (mütterliche) Risikofaktoren für die Entwicklung eines FASD bei Alkoholkonsum in der Schwangerschaft sind [2]:

- Alter $>30$ Jahre

- spezifische ethnische Zugehörigkeit

- geringer sozioökonomischer Status

- mütterliche Unterernährung, Mangel an Spurenelementen oder Vitaminen

- Stress

- Geburtshilfliche Komplikationen (z.B. Sauerstoffmangel bei der Geburt)

- Geschwister mit FASD (wird häufig mit jedem weiteren Kind ausgeprägter)

- Genetischer Hintergrund: FASD ist laut aktuellem Wissensstand nicht direkt erblich. Aber genetische
Faktoren können möglicherweise protektiv wirken oder die Vulnerabilität erhöhen. Epigenetische Studien an Tieren weisen darauf hin, dass in hohem Maße konsumierter Alkohol das Genom und damit auch die Spermien der Männer verändert. Hier sind die Studien, besonders an Menschen, aber noch nicht sehr weit.

\section{Was tun bei Verdacht auf FASD?}

Durch die richtige Diagnose zu einem frühen Zeitpunkt können Kinder oder Jugendliche mit Fetalen Alkoholspektrumstörungen frühzeitig adäquat gefördert werden. Eine Fehlbehandlung lässt sich vermeiden, Sekundärerkrankungen bzw. Komorbiditäten lassen sich reduzieren [4]. Und für die Eltern bedeutet es häufig eine Entlastung, wenn sie Gewissheit haben, was das Kind aufgrund der biologischen Schädigung des Gehirns leisten kann. Das kann auch das Kind entlasten, das merkt, dass es irgendwie anders ist.

Eine multidisziplinäre Leitliniengruppe entwickelte auf Basis einer umfangreichen evidenzbewerteten Literaturrecherche Empfehlungen für die Diagnostik der Fetalen Alkoholspektrumstörungen [3]:

- Fetales Alkoholsyndrom (FAS)

- Partielles Fetales Alkoholsyndrom (pFAS)

- Alkoholbedingte entwicklungsneurologische Störung (ARND)

- Alkoholbedingte angeborene Fehlbildungen (ARBD)

\section{INFO}

\section{Wirkung von Alkohol}

Die Entwicklung des Zentralen Nervensystems erfolgt über den gesamten Zeitraum der Schwangerschaft. Vereinfacht dargestellt, kann Alkohol sich folgendermaßen auswirken:

1. Trimenon: Die Zellen vermehren sich durch Zellteilung, es erfolgt bereits die erste Differenzierung. Wenn währenddessen eine Noxe wie Alkohol darauf einwirkt, teilen sich die Zellen nicht in ausreichendem Maße. Das Gehirn bleibt klein.

2. Trimenon: Die Zellen wandern vorprogrammiert zu den Orten ihrer Bestimmung, zum Beispiel im Cortex. Wirkt eine Noxe wie Alkohol darauf ein, kommt es zu Heterotopien. Korrekt gebildete und funktionierende Zellen gelangen nicht zu ihrer anatomisch üblichen Lokalisation und können die Funktion, die sie dort ausüben sollen, nicht ausführen.

3. Trimenon: Die Zellen differenzieren sich weiter, im Zentralnervensystem entstehen Synapsen, das neuronale Netzwerk baut sich auf. Wenn in diesem Stadium eine Noxe wie Alkohol darauf einwirkt, wird dieses wichtige Netz nicht vollständig ausgebildet. 
Die Leitlinie legt Kriterien fest, mit denen basierend auf vier diagnostischen Säulen Fetale Alkoholspektrumstörungen diagnostiziert werden können. Bei Verdacht auf FASD sollten Kinder zur weiteren Abklärung und Förderung an eine Institution mit Erfahrung in der Diagnose und Therapie Fetaler Alkoholspektrumstörungen (z.B. Sozialpädiatrisches Zentrum) weitergeschickt werden:

„Bei Kontakt zum Gesundheits- und Hilfesystem sollten, wenn ein Kind Auffälligkeiten in einer der 4 diagnostischen Säulen zeigt, die 3 anderen diagnostischen Säulen beurteilt oder ihre Beurteilung veranlasst werden. “ [3]

\section{Die vier diagnostischen Säulen sind:}

1. Wachstumsauffälligkeiten

2. Auffälligkeiten des Gesichtes

3. Auffälligkeiten des ZNS (funktionelle und strukturelle)

4. Mütterlicher Alkoholkonsum in der Schwangerschaft

\section{Diagnose Vollbild FAS}

Für die Diagnostik des Vollbildes des Fetalen Alkoholsyndroms müssen mindestens Kriterien der diagnostischen Säulen 1 bis 3 zutreffen:

\section{Wachstumsauffälligkeiten}

Zur Erfüllung des Kriteriums Wachstumsauffälligkeiten soll mindestens eine der folgenden Auffälligkeiten zutreffen:

- Geburts- oder Körpergewicht $\leq 10$. Perzentile

- Geburts- oder Körperlänge $\leq 10$. Perzentile

- Body-mass-Index $\leq 10$. Perzentile

Für sich alleine genommen sind Wachstumsauffälligkeiten ein relativ vages Kriterium, denn rund $10 \%$ der Kinder weisen diese auf. Doch in Zusammenhang mit Kriterien der anderen 3 diagnostischen Säulen ist es aussagekräftig. Die Beurteilung erfolgt adaptiert an das Alter bzw. Gestationsalter und Geschlecht. Die Dokumentation kann zu einem beliebigen Zeitpunkt erfolgen, d.h. das Kriterium ist genauso erfüllt, wenn ein Kind zu klein auf die Welt kommt und dann an Wachstum aufholt wie wenn es normalgewichtig zur Welt kommt und dann nicht altersgemäß zunimmt. Es sollte jedoch ausgeschlossen werden, dass die Wachstumsstörung allein durch andere Ursachen erklärt werden kann (s. Differentialdiagnostik).

\section{Faciale Auffälligkeiten}

Zur Erfüllung des Kriteriums Auffälligkeiten des Gesichtes sollen alle drei facialen Anomalien vorliegen, die ebenfalls zu einem beliebigen Zeitpunkt dokumentiert werden können:

- Kurze Lidspalten ( $\leq 3$. Perzentile): Zur objektiven Beurteilung der Lidspaltenlänge empfiehlt sich die Messung am Foto: Das Kind bekommt einen $\emptyset 1 \mathrm{~cm}$ Punkt auf die Stirn geklebt und wird fotografiert. Anhand 
FASD 4-Digit Diagnostic Code

๑ 2014 Susan Astley, University of Washington fasdpn.org
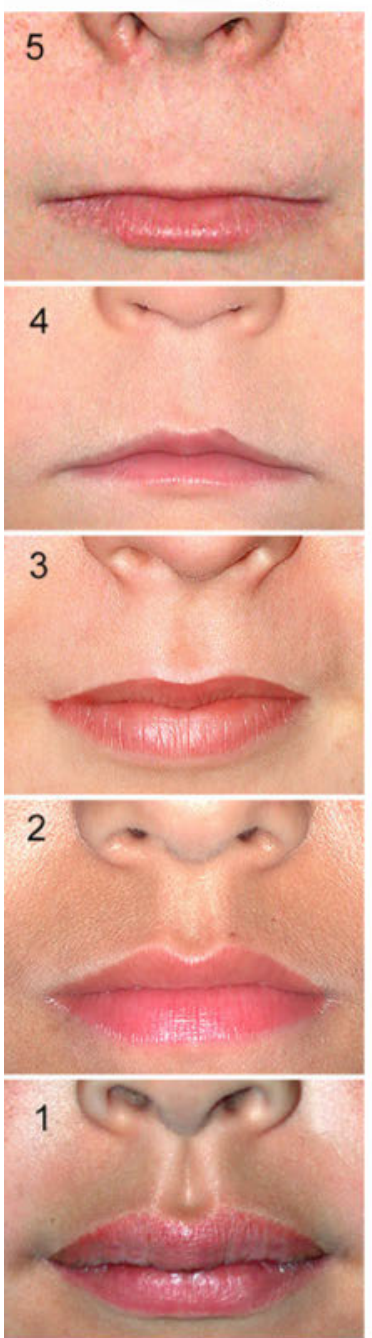

Lip-Philtrum Guide I
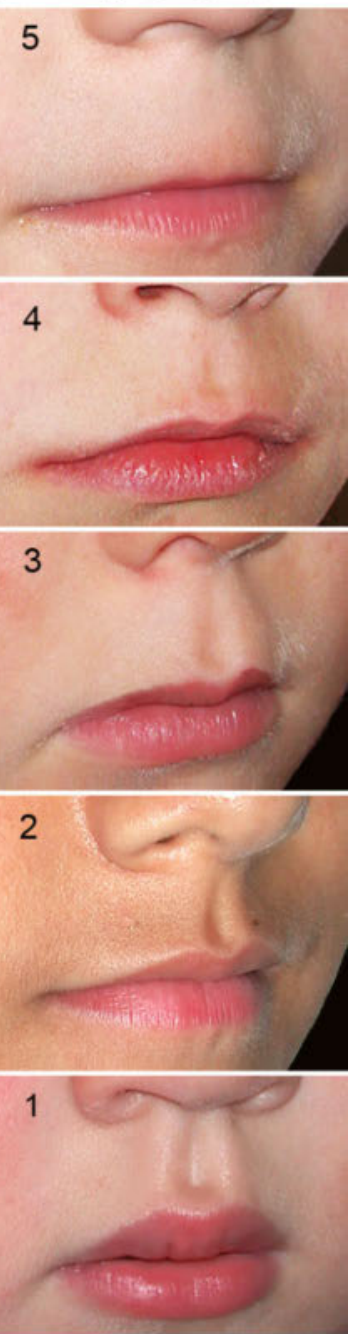

Philtrum Guide

For use as a digital image on a smartphone or tablet. Printing invalidates Guide.

Square ensures length by width ratio of image is correct.

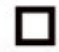

-Abb. 1 Der Lip-Philtrum-Guide der Universität Washington dient zur Beurteilung der Oberlippendicke und der Ausbildung des Philtrums. Das Philtrum besteht aus den zwei Falten und der Furche dazwischen, die sich zwischen Nase und Oberlippe befinden. Rang 3 entspricht der durchschnittlichen Ausprägung in der Normalbevölkerung. In Rang 4 und 5 sprechen eine charakteristisch dünne Oberlippe und ein verstrichenes Philtrum für FASD. Der Lip-Philtrum-Guide 1 (links) zeigt kaukasische, der LipPhiltrum-Guide 2 (nicht im Bild) afrikanische Ethnizität.

Freie digitale Bilder des Guides zur Nutzung auf Smartphone und Tablet erhältlich unter astley@uw.edu. Copyright 2017, Susan Astley PhD, University of Washington.

dieses Referenzmaßstabes wird mithilfe Foto nachgemessen, ob die Lidspaltenlänge unter der 3. Perzentile liegt. Achtung: Bitte keine Diagnostik nach dem Motto: „Das Kind hat Kulleraugen.“ Je nachdem, wie breit das Gesicht ist, wirken die Augen kürzer oder länger.

- Verstrichenes Philtrum: Die Falten zwischen Mund und Nase sind nicht vorhanden oder kaum ausgeprägt. Für die Beurteilung der Oberlippendicke und der Philtrumausprägung entwickelten Astley und Clarren (2000) den Lip-Philtrum-Guide mit Referenzfotos ( Abb. 1). Messungen mit vier oder fünf Punkten auf der Skala gelten als pathologische Ausprägungen von Philtrum und Oberlippe.

- Schmale Oberlippe (Rang 4 oder 5 Lip-Philtrum-Guide)

Diese drei facialen Screeningkriterien weisen eine Sensitivität von 99,8 \% auf (wenn das Kind ein FAS hat, zeigt es zu 99,8\% alle drei Auffälligkeiten) und eine Spezifität von 89 \% (89\% aller Kinder, die kein FASD haben, weisen hier auch nicht die Kombination aller drei Kriterien auf).

Bereits Neugeborene können faciale Auffälligkeiten aufweisen. Wenn zudem auffällt, dass der Kopfumfang zu klein und das Kind untergewichtig ist, sollte man die Diagnose FAS erwägen.

\section{ZNS-Auffälligkeiten}

Zur Erfüllung des Kriteriums Funktionelle ZNS-Auffälligkeiten sollte mindestens eine der folgenden Auffälligkeiten (1. oder 2.) zutreffen, die nicht altersadäquat ist und nicht allein durch den familiären Hintergrund oder das soziale Umfeld erklärt werden kann:

1. Globale Intelligenzminderung mindestens zwei Standardabweichungen unterhalb der Norm (IQ < 70) oder signifikante kombinierte Entwicklungsverzögerung bei Kindern unter zwei Jahren. Erfahrung aus eigener Praxis: 80 bis 90 \% der Kinder mit Vollbild FAS, die sich in unserer Ambulanz vorstellen, haben einen durchschnittlichen oder unterdurchschnittlichen IQ, aber keinen IQ unter 70.

Jedoch weisen sie mindestens drei Auffälligkeiten in folgenden Bereichen auf:

2. Leistung mindestens zwei Standardabweichungen unterhalb der Norm in mindestens drei der folgenden Bereiche (oder in mindestens zwei der folgenden Bereiche in Kombination mit Epilepsie):

- Sprache

- Feinmotorik

- räumlich-visuelle Wahrnehmung oder räumlich-konstruktive Fähigkeiten

- Lern- oder Merkfähigkeit

- exekutive Funktionen

- Rechenfertigkeiten

- Aufmerksamkeit

- soziale Fertigkeiten oder Verhalten

Für die Beurteilung der typischerweise betroffenen funktionellen ZNS-Bereiche geben in der Leitlinie vorgeschlagene neuropsychologische Testverfahren eine praxisbezogene Richtschnur [3]. 


\section{BEISPIEL}

Funktionelle ZNS-Auffälligkeiten im Alltag

Ein 12-jähriger Junge mit FASD will mit seiner Mutter einen Kuchen backen. Mehl und Eier fehlen. Die Mutter schickt den Jungen mit 20 Euro zum Supermarkt um die Ecke, zu dem sie immer gehen. Was dann passiert: Der Junge wird sehr bereitwillig den Auftrag annehmen. Das erste Problem: Er wird den Supermarkt nicht finden, obwohl er schon oft dort war. Grund: Er hat ein räumlich-visuelles Problem. Er findet sich nicht zurecht. Er wird sich verirren und nicht mehr wissen, wo er ist. Findet er den Supermarkt doch, wird er vergessen haben, was er kaufen soll. Hat die Mutter ihm einen Zettel geschrieben, ist nicht gesagt, dass er diesen findet. Im Supermarkt wird er nicht wissen, wo das Mehl steht und es z.B. im Kühlregal suchen. Er wird von dem Angebot, z.B. im Süßigkeitenregal, vollkommen abgelenkt sein. Falls er es doch schafft, nur Mehl und Eier zu kaufen, wird er der Kassiererin die 20 Euro geben und fragen: „Reicht das?" Er hat keine Idee von Mengen, kann nicht einschätzen, wie viel etwas wert ist oder kostet, kann nicht nachvollziehen, was er an Geld zurückbekommt. Diese Problematik hält sich bis ins Erwachsenenalter. Beim Einpacken wird er Eier unten in die Tasche legen, Mehl darauf fallen lassen, ein Teil der Eier geht dabei kaputt. Grund: Seine Exekutivfunktionen sind gestört. Höhere kognitive Prozesse wie ein Verständnis des Ursache-Wirkungs-Prinzips sind eingeschränkt. Er ist nicht fähig zur Handlungsplanung und -korrektur. Als er nach Hause gehen will, verläuft er sich wieder. Dann hält ein Auto neben ihm an, aus dem ein fremder Mann inn fragt, wohin er möchte. Wenn der Mann sehr freundlich anbietet, ihn mitzunehmen, kann es sein, dass der Junge einsteigt. Das Gefährdungspotenzial erkennt er nicht.

Zur Erfüllung des Kriteriums Strukturelle ZNS-Auffälligkeiten sollte außerdem folgende Auffälligkeit, adaptiert an Gestationsalter bzw. Alter und Geschlecht, dokumentiert zu einem beliebigen Zeitpunkt, zutreffen:

- zu kleiner Kopfumfang $\leq 10$. Perzentile / $\leq 3$. Perzentile

\section{Mütterlicher Alkoholkonsum}

Wenn Auffälligkeiten in den ersten drei diagnostischen Säulen vorliegen, kann die Diagnose auch ohne Bestätigung des Alkoholkonsums der Mutter während der Schwangerschaft gestellt werden, weil die anderen drei diagnostischen Säulen hoch sensitiv und spezifisch sind.

Die Leitliniengruppe definiert einen „wahrscheinlichen mütterlichen Alkoholkonsum während der Schwangerschaft" als mündliche oder schriftliche Angabe im Rahmen der Fremdanamnese [3].
Personen des privaten Umfeldes (unter Berücksichtigung möglicher familiärer Konflikte) können z.B. sein:

- Vater des Kindes

- während der Schwangerschaft mit der Mutter zusammenlebende/r Partner oder Partnerin

- andere Verwandte des Kindes

- andere Bezugspersonen, die in engem Kontakt zur Mutter stehen

Personen des beruflich-unterstützenden Umfeldes können z.B. sein:

- Hebammen

- betreuende Ärztinnen/Ärzte

- Fachkräfte der freien und öffentlichen Träger der Jugendhilfe (z.B. sozialpädagogische Familienhilfe)

\section{Zusammenfassung}

Im Säuglings- und Kleinkindalter hängt die Diagnose FAS von einer erfahrenen entwicklungsneurologischen Beurteilung ab. Im Kindes- und Jugendalter muss eine aufwendige psychologische Diagnostik erfolgen, da die Beeinträchtigung in funktionellen ZNS-Bereichen auch für die adäquate Förderung, das Funktionsniveau im Alltag und die Lebensqualität der betroffenen Jugendlichen und ihrer Familien entscheidend ist. Die Hebamme selbst kann keine Diagnose FASD stellen, sollte aber bei Auffälligkeiten des Kindes oder in der Familie den Verdacht äußern und eine weitere Beurteilung initiieren.

\section{Diagnose weiterer Fetaler Alkoholspektrumstörungen}

\section{Partielles Fetales Alkoholsyndrom (pFAS) [3]}

- Gleiche Auffälligkeiten des Gesichts, aber es müssen nur zwei der drei o.g. Auffälligkeiten auftreten

- Beeinträchtigung im ZNS in mindestens drei der oben genannten Bereiche (funktionell oder strukturell)

- Wichtig: Zur Diagnosestellung wird ein wahrscheinlicher oder bestätigter Alkoholkonsum der Mutter während der Schwangerschaft benötigt, weil die Spezifität abnimmt, wenn nicht alle drei facialen Auffälligkeiten vorliegen und die Wachstums-Säule wegfällt.

\section{Alkoholbedingte entwicklungsneurologische Störung (ARND) [3]}

Unter den diagnostischen Säulen fallen die Wachstumsauffälligkeiten und facialen Auffälligkeiten weg - es handelt sich um eine „nicht sichtbare Behinderung“. Dadurch ist die Diagnostik erschwert und es ist schwierig, die adäquate Förderung und Akzeptanz zu erreichen.

- Beeinträchtigung in mindestens drei der o.g. ZNS-Bereiche. Da es sich um eine nicht sichtbare Behinderung handelt und die Auffälligkeiten exakt bestimmt und immer zwei Standardabweichungen unter der Norm liegen müssen, muss eine 
ausführliche psychologische Diagnostik von einem FASD-erfahrenen Leistungserbringer erfolgen.

- Die Bestätigung des Alkoholkonsums der Mutter in der Schwangerschaft ist Voraussetzung für die Diagnose.

\section{Alkoholbedingte angeborene Fehlbildungen (ARBD)}

Wird als Diagnose heute in Deutschland nicht mehr gestellt, weil keine Evidenz für ARBD als eindeutige Krankheitsentität vorliegt und weil die Malformationen (z.B. Lippen-Kiefer-Gaumen-Spalte) nicht spezifisch sind (auch bei anderen Erkrankungen und bei ansonsten gesunden Kindern auftritt) [3].

\section{Warum eine Differentialdiagnostik wichtig ist}

Wachstumsdefizite, phänomenologische bzw. faciale Besonderheiten, Beeinträchtigungen in den für FASD typischen ZNS-Funktionsdomänen sollten immer klinischdifferentialdiagnostisch betrachtet werden. Differentialdiagnosen in allen Bereichen müssen medizinisch und psychologisch abgeklärt werden. Fehldiagnosen müssen aufgrund der Gefahr der sozialen Stigmatisierung weitmöglichst vermieden werden. Es muss aber bedacht werden, dass das Fehlen der Diagnose FASD häufig zu einer Überforderung des Kindes und seiner Bezugspersonen, zu einer deutlichen Belastung der Familien und zu frustrierenden Lebensverläufen führt. Dabei ist zu beachten, dass FASD sehr viel häufiger auftritt als andere Erkrankungen wie z.B. genetische Syndrome oder Stoffwechselerkrankungen.

Im Rahmen der Differentialdiagnose sollte ausgeschlossen werden, dass sich die festgestellte Wachstumsstörung durch andere Ursachen erklären lässt, z.B. familiärer Kleinwuchs (kleine Eltern, kleine Kinder), konstitutionelle Entwicklungsverzögerung, pränatale Mangelzustände, Skelettdysplasien, hormonelle Störungen, genetische Syndrome (z.B. Turner-, Silver-Russel-Syndrome), chronische Erkrankungen, Malabsorptionen, Mangelernährung, Vernachlässigung [9].

Bei den facialen Auffälligkeiten ist auszuschließen, dass diese zurückzuführen sind z.B. auf toxische Effekte in der Schwangerschaft (z.B. Antikonvulsiva, Tulol, maternale Phenylketonurie) oder genetisch bedingte Erkrankungen (z.B. Di-George-Syndrom, Noonan-Syndrom) [9].

Bei den funktionellen ZNS-Auffälligkeiten sind verschiedene psychische Störungen, andere Entwicklungsstörungen und z.B. Suchterkrankungen auszuschließen. Bei den strukturellen Störungen ist z.B. auszuschließen, dass es sich um familiäre Mikrocephalie, genetische Syndrome, pränatale Mangelversorgung, Infektion, hypoxischischämische Hirnschädigung, maternale Erkrankungen, postnatale Mangelernährung, Stoffwechselstörungen oder chronische Erkrankungen handelt [9].

\section{Prognose FASD}

61\% der Erwachsenen mit FASD haben ihre Schullaufbahn abgebrochen [4]. Lediglich 13\% der Erwachsenen mit FASD hatten einmalig einen Job auf dem ersten Arbeitsmarkt. Nur ca. 1/3 der erwachsenen Patienten mit FASD konnten ein selbstständiges Leben führen, 2/3 lebten betreut - in Institutionen oder auch im Erwachsenenalter noch mit den Eltern [5]. 67\% der Betroffenen mit FASD berichteten über erlebte körperliche oder sexuelle Misshandlung und 35\% hatten selbst ein Alkohol- oder Drogenproblem. 60\% der Erwachsenen mit FASD gerieten in Gesetzeskonflikte [4].

\section{Therapie}

Die FASD resultiert aus einer alkoholtoxischen Gehirnschädigung, die biologisch nicht reparabel ist. Eine frühzeitige Diagnostik und ein stabiles förderndes Umfeld kann den Langzeit-Outcome der Kinder mit FASD jedoch deutlich positiv beeinflussen [4]. Im Rahmen der Therapie kann funktionell mit den Kindern und Jugendlichen gearbeitet werden und sie können individuell in ihrer Entwicklung unterstützt werden. Therapieziel ist die Verbesserung der Alltagsfunktionen der Betroffenen und der Lebensqualität der Familien. Die Therapie sollte wegen der Symptomvielfalt von Patienten mit dem Spektrum alkoholbedingter pränataler Schädigungen nicht diagnose- sondern symptomorientiert ausgerichtet sein und die Bezugspersonen sind in Form von Trainings und Beratung immer mit einzubeziehen [6].

Für die frühe Diagnose sind auch Hebammen gefragt: Bereits Neugeborene können die facialen Auffälligkeiten aufweisen. Auch wenn der Kopfumfang zu klein und das Kind untergewichtig ist, sollte an FAS gedacht werden und der Verdacht auf FASD in einem spezialisierten Zentrum überprüft werden.

\section{Pocket-Guide}

Einen FASD-Pocket-Guide mit einer Übersicht aller diagnostischen Kriterien für Fetale Alkoholspektrumstörungen finden Sie zum Download unter www.ispz-hauner.de unter der Rubrik > Abteilungen > TESS - FASD

Die Leitlinie zur Diagnose der FASD steht zum Download unter www.awmf.org/leitlinien/detail/I/022-025.html

\section{Literatur}

[1] GEDA - Studie zur Gesundheit in Deutschland des Robert Koch Instituts. (2012). Retrieved October 26, 2015, from http://www.rki.de/DE/Content/Gesundheitsmonitoring/ Gesundheitsberichterstattung/GBEDownloadsB/GEDA12.pdf

[2] Landgraf MN, Hilgendorff A, Heinen F (2014). Mütterlicher Alkoholkonsum in der Schwangerschaft und fetales Alkoholsyndrom. Monatsschrift Kinderheilkunde; 10: 903-910.

[3] Landgraf MN, Heinen F. Fetale Alkoholspektrumstörungen - FASD Diagnostik in der Kinder- und Jugendmedizin. 
Praxisguide der S3 Leitlinie. Monatsschrift Kinderheilkunde 2016. ePub ahead of print Oct 2016: http://link.springer. com/article/10.1007/s00112-016-0191-y

[4] Streissguth AP et al. (2004). Risk Factors for Adverse Life Outcomes in Fetal Alcohol Syndrome and Fetal Alcohol Effects. Journal of Developmental and Behavioral Pediatrics, $25,228-238$.

[5] Spohr HL, Steinhausen HC. (2008). Fetal alcohol spectrum disorders and their persisting sequelae in adult life. Deutsches Aerzteblatt International, 105, 693-8.

[6] Landgraf MN, Giese RM, Heinen F. Fetale Alkoholspektrumstörungen - Diagnose, neuropsychologische Testung und symptomorientierte Förderung. Z Kinder Jugendpsychiatr Psychother. 2017;45:104-117

[7] May PA, Fiorentino D, Gossage PJ et al. Epidemiology of FASD in a province in Italy: Prevalence and characteristics of children in a random sample of schools. Alcohol Clin Exp. 2006; Res 30:1562-75

[8] May PA, Fiorentino D, Coriale G et al. Prevalence of children with severe fetal alcohol spectrum disorders in communities near Rome, Italy: new estimated rates are higher than previous estimates. Int J Environ Res Public Health. 2011; 8:2331-51

[9] S3-Leitlinie Diagnose der Fetalen Alkoholspektrumstörungen. Downloadbar unter: http://www.awmf.org/leitlinien/ detail/II/022-025.html
Autorinnen/Autoren

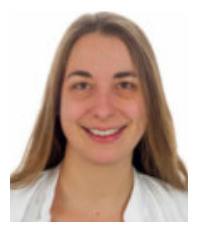

Dr. med. Dipl.-Psych. Mirjam N. Landgraf

ist Fachärztin für Kinder- und Jugendmedizin, Schwerpunkt Neuropädiatrie, Diplompsychologin, Oberärztin, Leiterin der TESS-Ambulanz im iSPZ Hauner und Leitlinienkoordinatorin FASD.

\section{Korrespondenzadresse}

Ludwig-Maximilians-Universität München

Dr. von Haunersches Kinderspital

Abteilung für Pädiatrische Neurologie, Entwicklungsneurologie und Sozialpädiatrie

Lindwurmstr. 4

80337 München

E-Mail: mirjam.landgraf@med.uni-muenchen.de 